\title{
Contenidos de Anatomía en Diseños de Aprendizaje Dispuestos en LAMS e Integrado a Moodle
}

\author{
Anatomy Contents in Learning Designs Displayed in LAMS and Integrated to Moodle
}

\author{
"Sandra Bucarey A.; **Erick Araya A.; *** Ximena Cabezas O. \& ** Luis Álvarez G.
}

BUCAREY, A. S.; ARAYA, A. E.; CABEZAS, O. X. \& ÁLVAREZ, G. L. Contenidos de anatomía en diseños de aprendizaje dispuestos en LAMS e integrado a Moodle. Int. J. Morphol., 29(2):363-370, 2011.

RESUMEN: Este trabajo trata la experiencia del uso de LAMS en procesos de aprendizaje e-learning para el área de la salud. Se trabajó con contenidos de anatomía del hígado humano, siguiendo estándares educativos y metodologías necesarias para crear Diseños de Aprendizaje (LD) y recursos educativos de calidad e interoperables. El Instituto de Anatomía, Histología y Patología fue responsable del diseño e implementación de Objetos de Aprendizaje (OAs) en anatomía de hígado (con Macromedia flash), mientras el Instituto de Informática se encargó de abordar la gestión y proceso del recurso pedagógico, integrando un especialista (LAMS y Moodle) para el diseño y gestión de aprendizaje. LAMS fue usado para crear actividades de aprendizaje basado en LD integrado a Moodle, como parte de un curso de estudiantes de medicina. La encuesta de satisfacción aplicada a estos estudiantes mostró, que casi el 70\% de ellos consideró las actividades y objetivos comprensibles y suficientemente detallados, que las evaluaciones fueron coherentes con los contenidos y que existe aprendizaje colaborativo. Así mismo el 67\% aseguró que volvería a usar un plan de lección en esta modalidad y sólo un 33\% lo haría ocasionalmente.

PALABRAS CLAVE: Diseños de Aprendizaje, Objetos de Aprendizaje, Anatomía humana, LAMS, Moodle.

\section{INTRODUCCIÓN}

Desde el 2003 se han estado desarrollando OAs como recursos reutilizables para la enseñanza de la anatomía humana. Dado que el OA es considerado-conceptualmentecomo una unidad independiente de contenido, puede ser usado en diferentes contextos de aprendizaje. Esto es importante para dar a un recurso digital el carácter de OA, con énfasis en la reutilización y con una apropiada metadata para su identificación. El uso adecuado de un OA que ayude a la comprensión del hígado humano se adapta mejor a los nuevos paradigmas basados en conocimiento y aprendizaje colaborativos. Esto es un cambio en la generación de las herramientas educacionales, pasando desde lo unidireccional a lo bidireccional y también a lo social. Si más personas están involucradas en la construcción de recursos digitales y si éstas, en algunos casos, son estudiantes involucrados en el proceso, sería posible lograr un aprendizaje más efectivo. Además, este trabajo introduce un nuevo enfoque para la organización de los OAs y su secuenciación en actividades de aprendizaje en el área de la salud, los Learning Design
(Diseños de Aprendizaje, LD de ahora en adelante), (Koper \& Tattersall, 2005; IMS Global Learning Consortium Inc., 2003), estructura que se enmarca en los principios de la educación virtual.

La Facultad de Medicina de la Universidad Austral de Chile, dicta siete carreras de pregrado (Medicina, Odontología, Enfermería, Obstetricia, Tecnología Médica, Kinesiología y Terapia Ocupacional); cuatro diplomados; tres magíster; y un doctorado en Ciencias Médicas. Hasta ahora el pregrado es completamente presencial y se trabaja informalmente semipresencial en el desarrollo de algunas unidades de cursos de postgrado. Se prevé que a corto plazo, la universidad generará las políticas necesarias para el desarrollo de la educación virtual. Por otra parte, el Grupo de Investigación en Tecnologías de Aprendizaje GITA, conformados por investigadores tanto de la Facultad de Medicina como del Instituto de Ciencias de la Ingeniería, ha desarrollado y validado numerosos OA's para el área de la salud.

\footnotetext{
* Instituto de Anatomía, Histología y Patología. Facultad de Medicina, Universidad Austral de Chile, Valdivia, Chile.

*** Instituto de Informática. Facultad de Ciencias de la Ingeniería, Universidad Austral de Chile, Valdivia, Chile.

*** Instituto de Medicina. Facultad de Medicina, Universidad Austral de Chile, Valdivia, Chile.
} 
Los OAs han sido muy demandados durante los últimos años. Para estandarizar sus objetivos y clasificarlos adecuadamente, se definió una especificación basada en una estructura de metadatos (IEEE LOM, 2002). Con ello se añadió valor a los OA's, en el sentido de facilitar su reutilización. Sin embargo, esa especificación no es suficiente para justificar un aprendizaje efectivo. En ese contexto los LD, que incluyan en sus secuencias a OA's, son mucho mejores para potenciar el aprendizaje.

Este trabajo usa específicamente el OAAnatomía del hígado humano, que fue construido en el área de anatomía del Instituto de Anatomía, Histología y Patología de la Universidad Austral de Chile.

\section{MATERIAL Y MÉTODO}

Objeto de Aprendizaje: Anatomía del Hígado Humano. El OA incorporado en el LD, ha sido usado desde el 2004 y actualizado para enseñar en diferentes cursos de anatomía (Bucarey \& Álvarez, 2004). Con la incorporación del LD, se puede usar este OA probado, siguiendo la secuencia lógica para crear actividades de aprendizaje en LAMS.

La enseñanza tradicional de la anatomía del hígado humano, generalmente se subdivide en 5 componentes:

Situación y Ubicación; Estructura Externa; Segmentación Hepática; Vías Biliares; Vasos y Nervios. Además se incluyen tres componentes adicionales:
1. Webgrafía: Para revisar en Internet más información o diversos procedimientos quirúrgicos o de disección, todo vinculado al tema central del OA. Con esta herramienta un estudiante puede aprender más acerca del hígado humano, utilizando enlaces a sitios web recomendados por el mismo recurso, material que ha sido revisado y seleccionado por su contenido pedagógico y multimedial.

2. Imagenología: Se muestra el hígado humano a través de diversos recursos imagenológicos.

3. Imágenes de Pabellón. Presenta fotografías del hígado tomadas por los estudiantes y personal del área de anatomía.

Cada sección ha sido mejorada año tras año, con la participación colaborativa de estudiantes de algunas clases. Las conclusiones de esta interesante metodología donde los estudiantes son parte de su propio aprendizaje fue presentado en XXVI Congreso Chileno de Anatomía VII Congreso de Anatomía del Cono Sur (Bucarey \& Álvarez, 2006). La Fig. 1 muestra el menú principal del OA, con cada una de sus secciones. El OA fue empaquetado bajo SCORM.

Los primeros cinco componentes de este OA fueron subdivididos en cinco OAs más pequeños, para construir LD para estudiantes de Medicina, Enfermería y Obstetricia. Esto, porque son diferentes especialidades (Medicina, Enfermería y Obstetricia) con diferentes objetivos de aprendizaje.

Todos los OAs fueron usados en el LD para estudiantes de Medicina. Sólo los OAs de Situación y Ubicación, Estructura Externa y Vías Biliares fueron usados para Enfermería y Obstetricia.

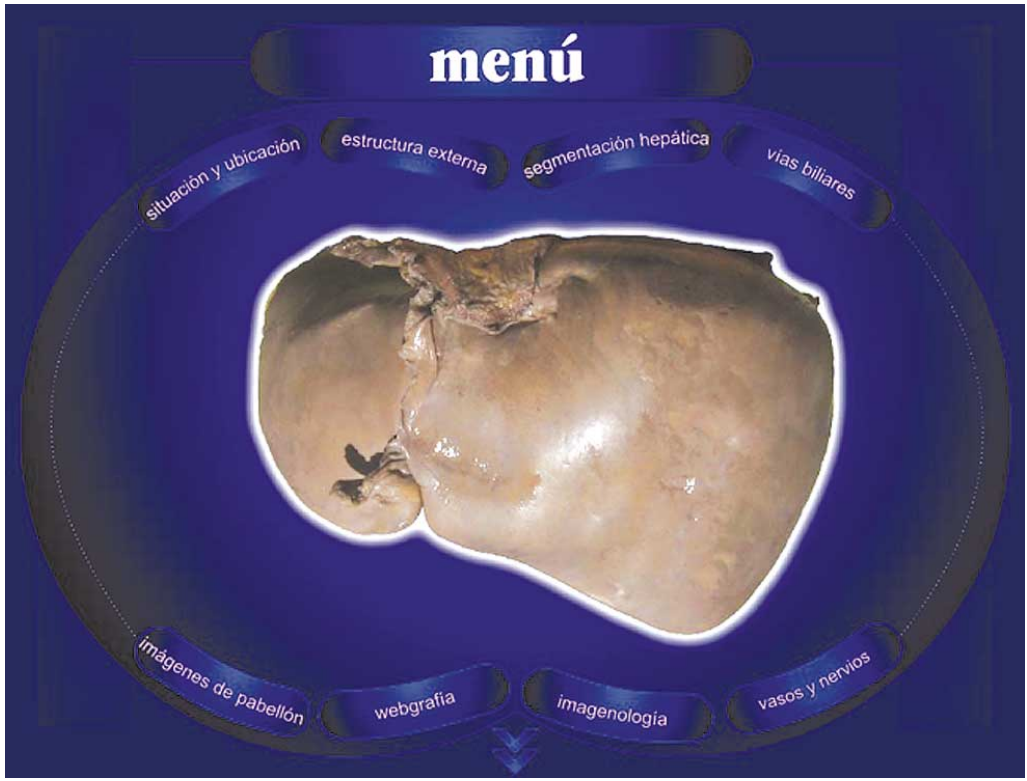

Fig. 1. Menú principal del OA anatomía del hígado humano.
Una Metodología para Construir Diseños de Aprendizaje. En general, el procedimiento usado para construir un LD es: el profesor hace un plan de lección como un texto narrativo, analiza los módulos para disponerlos en LAMS y finalmente construye la secuencia.

Plan de Lección. Un plan de lección es como un guión, preparado para enseñar una lección específica. El plan de lección consta de dos secciones principales: la metadata y el contenido. Usualmente, la metadata específica el título, autor, roles (aprendices y personal), objetivos de aprendizaje, requerimientos, materiales, etc. El contenido es, por lo general, dividido en actos, algunos ejemplos pueden ser encontrados en http://www.lessonplanz.com/. 
Tabla I. Plan de Lección para estudiantes de la Carrera de Medicina.

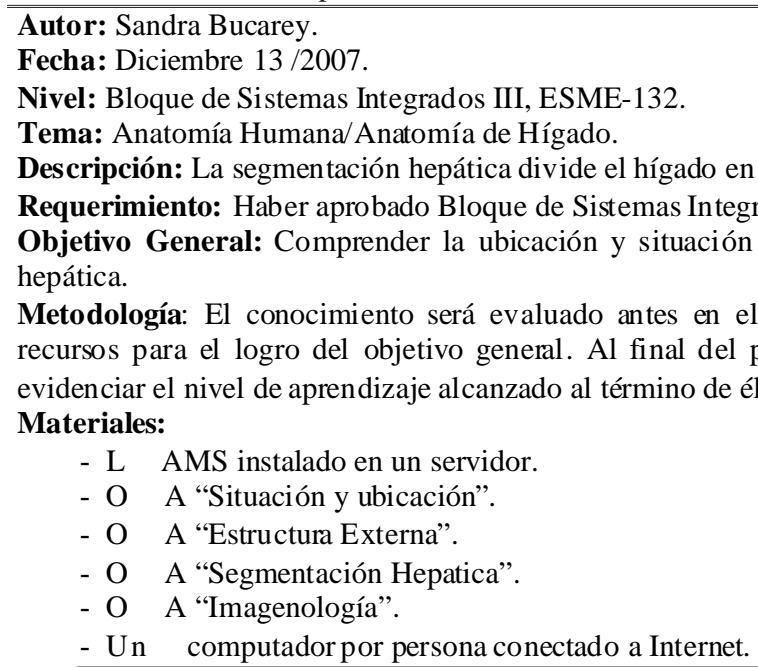

\section{Primer Acto.}

1. El profesor explica la metodología y hace una introducción donde habla del sistema digestivo y del hígado como componente de éste.

2. Estos temas son importantes para la compresión de la segmentación hepática porque éste es un órgano que puede ser trasplantado en forma completa o parcialmente. Una persona puede vivir con sólo una parte de su hígado gracias a la estructura segmentada de este.

3. Se informará que como primera etapa del curso, es necesario llevar a cabo una evaluación diagnóstica para que cada alumno compruebe sus avances al final del curso.

Segundo Acto

Responder la prueba de diagnóstico. Disponible en el archivo adjunto "Test de diagnóstico MED.doc"

Tercer Acto

Dispone de las siguientes actividades opcionales para que sean llevadas a cabo por los estudiantes.

1. Revisar el OA "Situación y Ubicación".

2. Revisar el OA "Estructura Externa".

3. Revisar el OA "Segmentación Hepática”.

4. Revisar el OA "Imagenología".

Cuarto Acto

Participar en el foro: Implicancia clínica de la segmentación hepática.

El foro comienza con la siguiente pregunta a debatir. ¿Cuáles son los componentes responsables de la segmentación hepática y cómo dividen éstos al hígado?

Quinto Acto

Enviar al profesor un breve texto con las conclusiones obtenidas debatidas en el foro.

Sexto Acto

Responder a la prueba de alternativas disponibles en el archivo adjunto "PREGUNTAS ANATOMIA HIGADO HUMANO I MED.doc"

Séptimo Acto

Análisis de la evaluación diagnostica y corrección de errores, comparando el nivel inicial con el nivel alcanzado al final del curso.

Condición:

Si los resultados del test no son satisfactorios, entonces el estudiante debe revisar el OA correspondiente para responder el test nuevamente.

Diseños de Aprendizaje propuestos. Tres LD han sido desarrollados, dos para las carreras de Enfermería y Obstetricia y uno para Medicina. La Tabla I, muestra el plan de lección para la carrera de medicina.

Implementación de los Diseños de Aprendizaje en LAMS. LAMS es un secuenciador de actividades de aprendizaje muy completo y de código abierto, que pone énfasis en el proceso de aprendizaje más que en el contenido, lo que se adapta con el objetivo de crear actividades pedagógicas.

Para implementar un LD usando LAMS, se desarrollaron tres OA's, diseñados a partir del OA de anatomía del hígado humano. La Fig. 2, muestra la secuencia de aprendizaje en LAMS para los estudiantes de Medicina. Esta secuencia es la implementación del plan de lección mostrado en la Tabla I. 


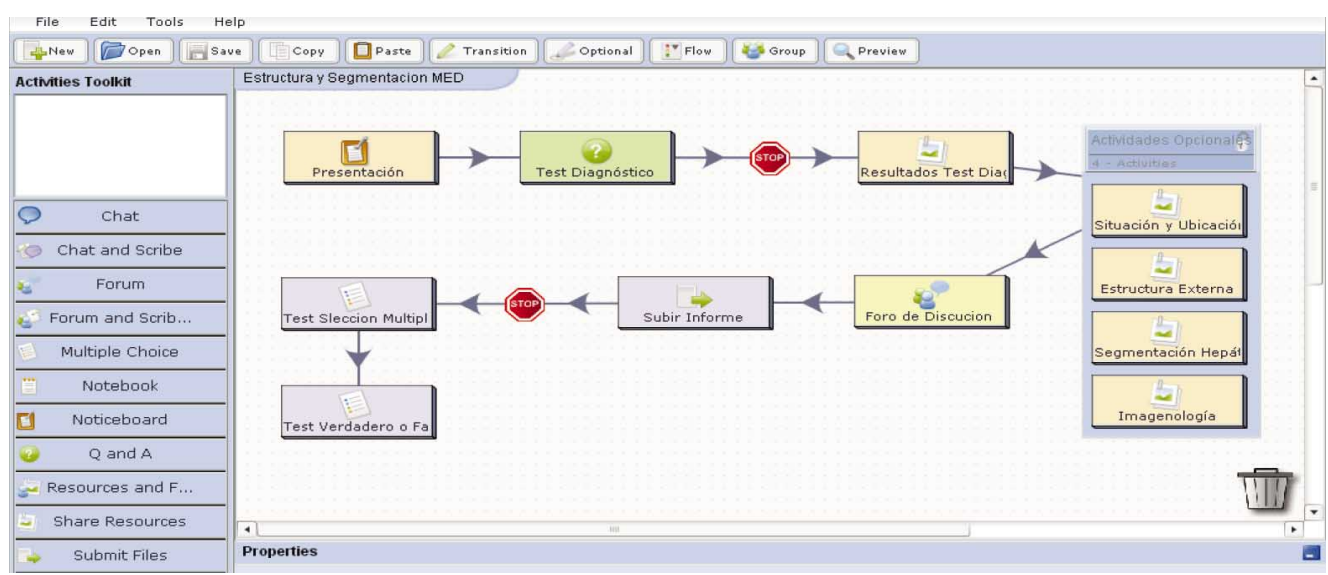

Fig. 2. Versión LAMS para la estructura y segmentación del hígado humano en un LD para la carrera de Medicina.

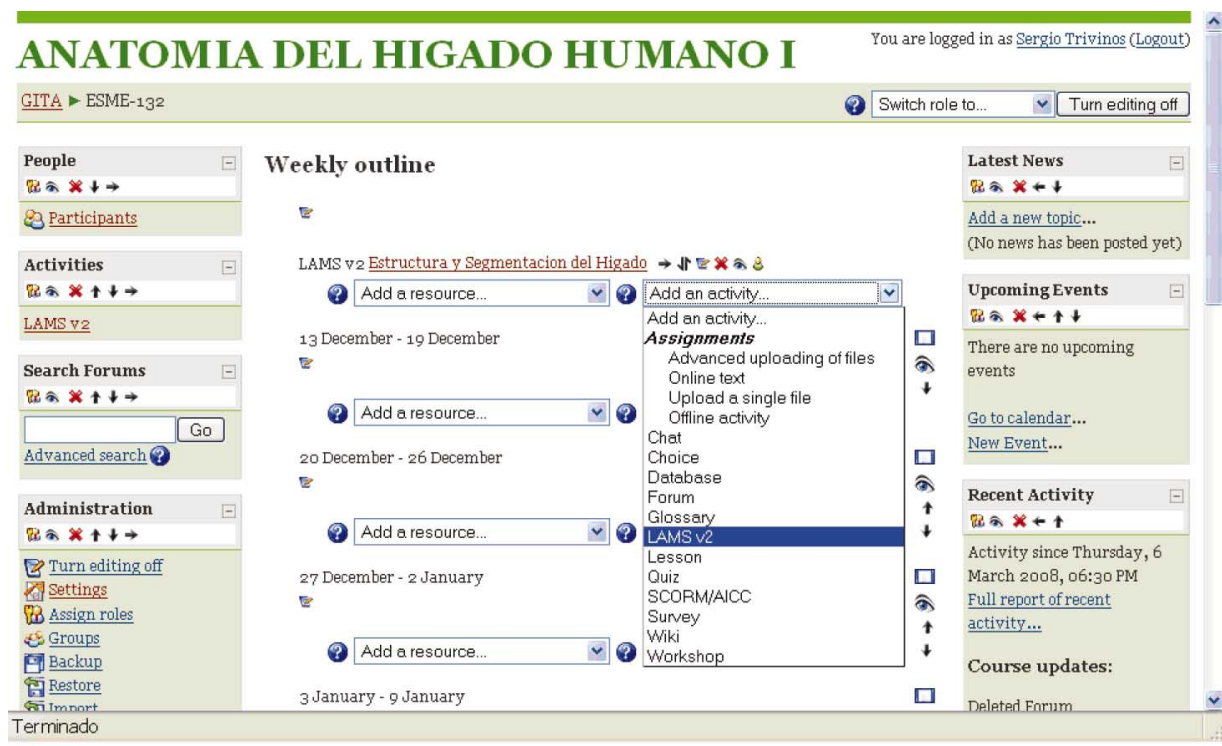

\begin{tabular}{|c|c|}
\hline ANATOMIA DEL HIGADO HUMANO I & You are logged in as Sergio Trivinos (Logout) \\
\hline GITA ESME-1,32 & \\
\hline
\end{tabular}

LAMS v2 Adding a new LAMS v20

Sequence

The directory structure below contains the sequences you can create a lesson for. Select one and click on the next button to continue.

My Workspace

由-gsergio Trivinos

DEstructura y Segmentacion MED

由]MY Groups

Create sequence Refresh workspace Cancel Next

(i) Moodle Docs for this page

You are logged in as Sergio Trivinos (Logout)

ESME-132

Terminado

Fig. 3. Pantallas usadas por los profesores para construir un LD en LAMS usando Moodle. 
Todos los estudiantes deben completar la prueba diagnóstico para revisar los OA como una secuencia; un módulo STOP fue incluido entre los módulos "Presentación” y "Test Diagnóstico". Otro módulo STOP es usado entre "Subir Informe" y "Test de Selección Múltiple".

Las actividades o lecciones comunes habituales son complementadas con los LD's.

Uso de Moodle para Ejecutar Actividades de Aprendizaje de LAMS. El LMS Moodle tiene la posibilidad de incorporar un gran número de módulos adicionales y plug-ins (ver http://moodle.org/). Por otra parte, LAMS usa OA's sólo en un módulo (comparte recursos). Para los usuarios (estu- diantes y profesores), Si un curso tradicional ya se encuentra en una plataforma tipo Moodle y el profesor requiere además incorporar LD's, la mejor solución es usar Moodle y LAMS integrados (Fig. 3).

Algunas ventajas para usar LAMS integrado a Moodle son:

1. El usuario administrador es solo uno. El módulo de administración de usuarios de Moodle tiene más funcionalidades que algún módulo en LAMS. De esta forma es suficiente una cuenta por cada usuario.

2. Toma todas las ventajas de LAMS y Moodle solo en una plataforma.

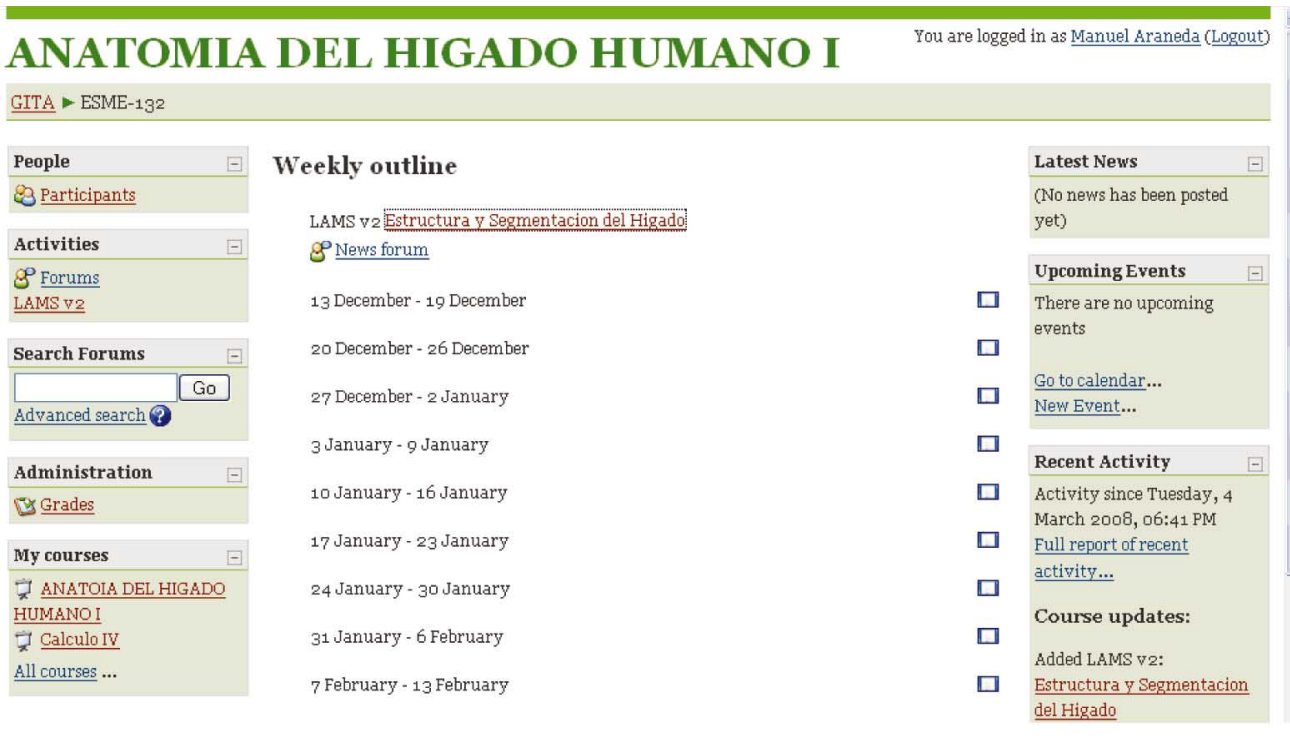

ANATOMIA DEL HIGADO HUMANO I

GITA ESME-132 $\rightleftharpoons$ Estructura y Segmentacion del Higado

Estructura y Segmentacion del Higado

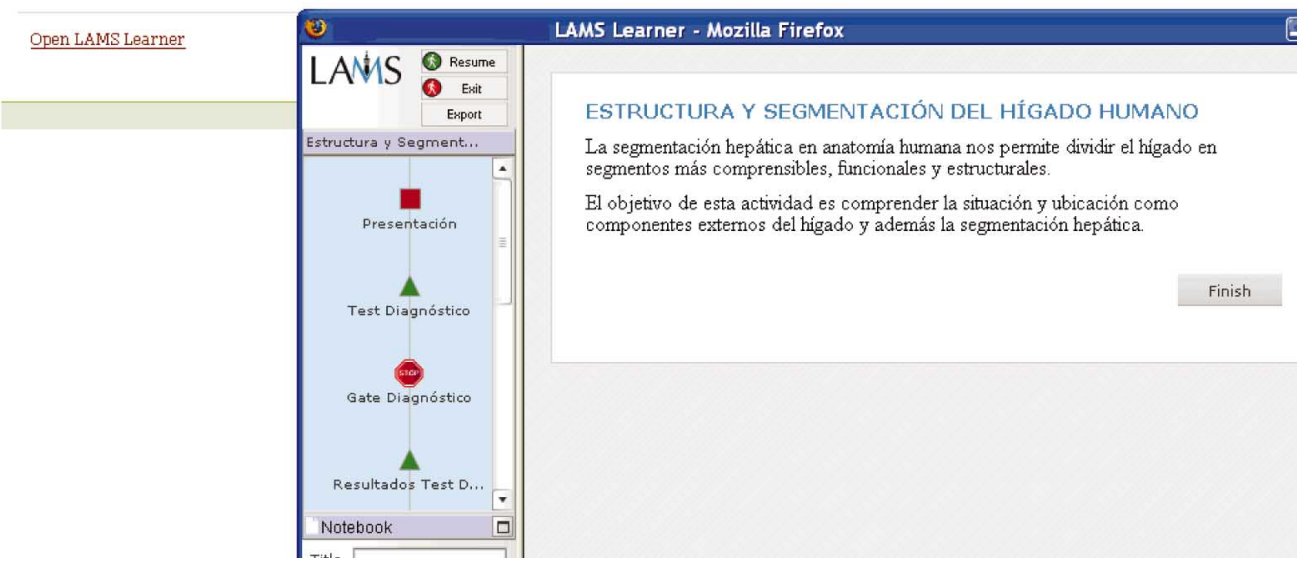

Fig. 4. Pantalla usada por los estudiantes para usar un LD en LAMS usando Moodle. 
3. Para estudiantes y profesores es más fácil usar una sola plataforma.

4. Ambas plataformas son de código abierto. En particular, Moodle tiene una buena evaluación, (The Open Polytechnic of New Zealand, 2004).

La Fig. 4 muestra pantallas disponibles para los estudiantes, cuando acceden a un LD (usando LAMS) a través de Moodle.

La ventaja es que el estudiante puede hacer uso de esta actividad en cualquier lugar, por ejemplo en su casa, complementando lo aprendido en clases. Por otra parte, los profesores del curso pueden acceder, en modo Monitor, a la evolución del estudiante en el proceso de aprendizaje del hígado humano, en cualquier momento que deseen, e intervenir en ese proceso, mediante recursos como chat o foros, disponibles por la actividad LD. Se potencia, de ese modo, el aprendizaje virtual de la actividad.

\section{RESULTADOS}

El año académico en la Universidad Austral de Chile comienza en marzo, y el LD fue usado con estudiantes de diferentes carreras después de la fecha mencionada.

Al finalizar por completo la experiencia de estudio a través del LD, a los estudiantes de las escuelas de Obstetricia, Medicina y Enfermería, involucrados por pri- mera vez en un proceso como éste, se les solicitó contestar una encuesta de satisfacción, de carácter técnico-pedagógico y aplicable a cualquier LD. Cada una de las respuestas se clasificó en una de 4 categorías: Muy de acuerdo - De acuerdo - En desacuerdo y Muy en desacuerdo.

Los resultados de las respuestas ante las preguntas “ ¿Se especifican adecuadamente las actividades con su tiempo de duración?” y “¿Los objetivos del plan de lección son comprensibles y dejan claro qué serán capaces de hacer los estudiantes una vez terminada la lección?" Se muestran en la Figura 5, donde es clara la tendencia hacia la opción Muy de acuerdo.

De igual modo, los resultados de las respuestas ante las preguntas "¿Las actividades del plan de lección son las adecuadas para permitir a los estudiantes alcanzar los objetivos planteados?” y “¿Existe aprendizaje colaborativo, es decir, los estudiantes construyen su conocimiento interactuando con otros estudiantes de su misma clase?" se muestran en la Fig. 6.

Para la pregunta " $¿$ Los recursos educativos sobre hígado son ad-oc a los objetivos planteados y el tipo de estudiante?, el $100 \%$ lo considera muy adecuado. Finalmente, para la pregunta con categorías Nunca, Ocasionalmente, Varias veces y Siempre, “¿Volvería a usar un plan de lección en LAMS para el aprendizaje de asignaturas que debe cursar?", el 67\% afirma que Siempre y el restante $33 \%$ responde Ocasionalmente.
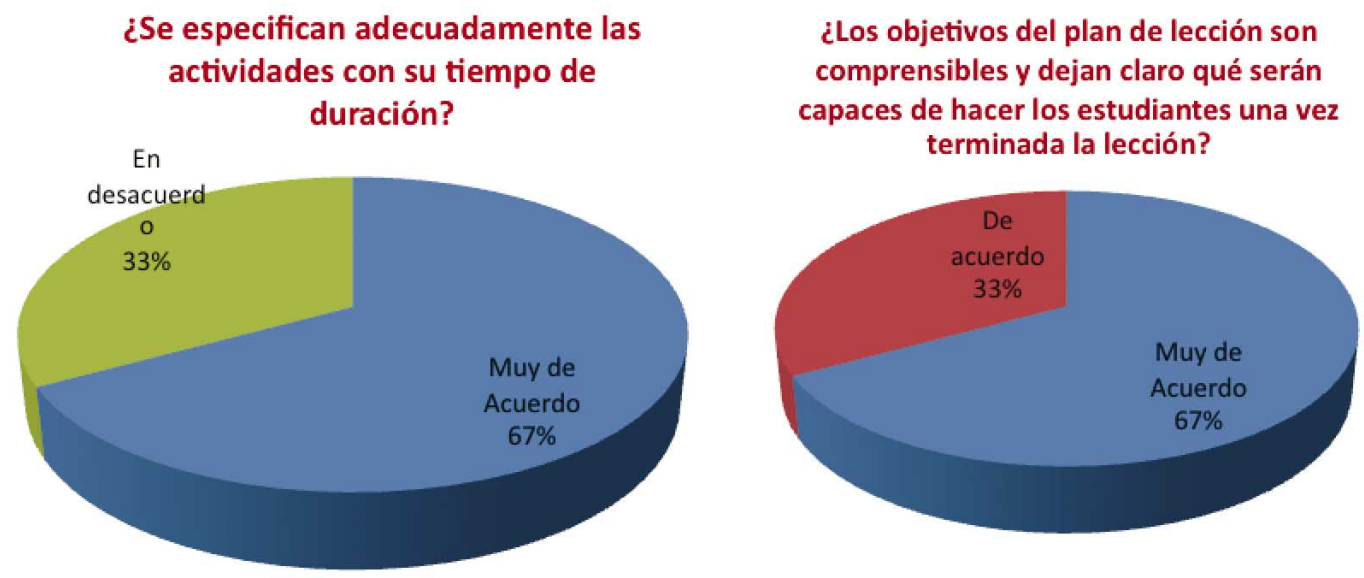

Fig. 5. Respuestas de estudiantes con clara tendencia hacia la opción "Muy de acuerdo". 

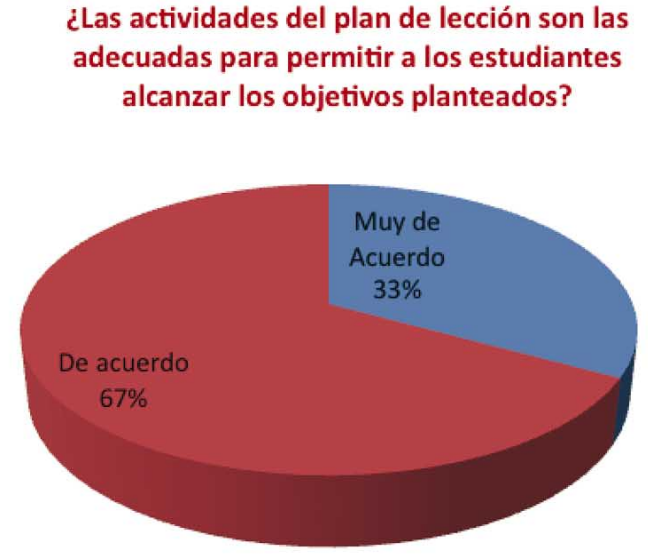

\section{"¿Existe aprendizaje colaborativo, es decir, los estudiantes construyen su conocimiento interactuando con otros estudiantes de su misma clase?}

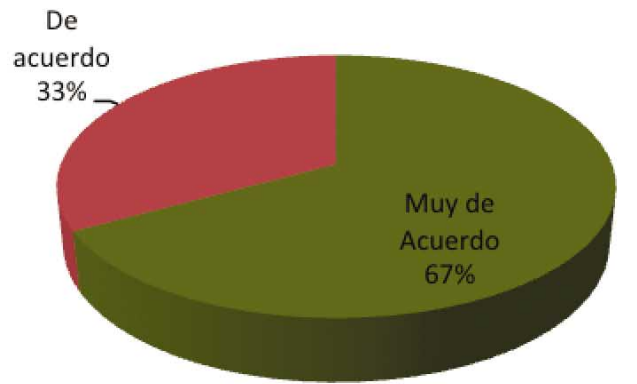

Fig. 6. Respuesta de los estudiantes frente a las actividades con TIC, que indican tendencia a estar "De acuerdo" o "Muy de acuerdo"

\section{DISCUSIÓN}

Para la implementación de un LD se recomienda comenzar por los planes de lección, especialmente cuando los conceptos de LD no son familiares para los profesores y pueden ser usados como un complemento de lecciones comunes en actividades presenciales o adoptados completamente para la enseñanza en modalidad semipresencial o virtual.

Una vez implementado el LD se hace fácil la modificación para otros cursos: Hacer un LD es un trabajo pesado y ocupa mucho tiempo construirlo por primera vez, después se puede ir modificando de acuerdo a los requerimientos de un curso. Por otra parte, la incorporación de OAs, construidos con colaboración de estudiantes, transforma al LD en un potente recurso para el aprendizaje, lo que se aprecia al constatar que casi al $70 \%$ de estudiantes que vivenciaron por primera vez un proceso de enseñanza aprendizaje siguiendo un LD volverían a usar esta modalidad. En este resultado se debe considerar también, que para los investigadores de este proyecto, fue una primera experiencia de aplicación con estudiantes y de especial impacto por ser usada en el área de la salud.

En base a lo anterior, para los usuarios de Moodle es fácil construir LD en Moodle- LAMS integrados, que solo en Moodle porque ellos ven LAMS como algún módulo de Moodle.

Finalmente, al analizar las respuestas de la encuesta de satisfacción, se concluye que los estudiantes valoran la incorporación de LD en el aprendizaje de la anatomía sobre estas plataformas educativas. La totalidad de ellos manifestó que los recursos educativos sobre la anatomía del hígado humano, son adecuados para los objetivos planteados y que volverían a usar un plan de lección en LAMS para el aprendizaje de otras asignaturas a cursar.

Se espera seguir construyendo OA's del área de la Salud para incorporarlos en LD de mayor tamaño, es decir correspondientes a unidades completas de cursos que se dictan con contenidos de anatomía, en especial con los sistemas digestivo y urinario que ya cuentan con material elaborado. La construcción de OA's se realizará siguiendo la metodología colaborativa descrita en el Manual de Construcción Colaborativa de Objetos de Aprendizaje para el Área de la Salud (Bucarey et al., 2010), dada la experiencia y buena evaluación alcanzados y descritos en el mismo libro.

\section{AGRADECIMIENTOS}

Los autores agradecen el apoyo de la Dirección de Investigación y Desarrollo (DID) de la Universidad Austral de Chile a través de sus proyectos No. S-2007-15 titulado Diseño de aprendizaje y Gestión en el aula; también por el proyecto DID No S-2006-30 titulado Actividades de Aprendizaje con contenidos Anatomoclínicos bajo la Especificación IMS-LD, y, finalmente, al proyecto DID $\mathrm{N}^{\circ} \mathrm{S}$ 2007-02. Así mismo, los autores agradecen a los miembros del Grupo de Investigación en Tecnologías de Aprendizaje (www.gita.cl). 
BUCAREY, A. S.; ARAYA, A. E.; CABEZAS, O. X. \& ÁLVAREZ, G. L. Anatomy contents in learning designs displayed in LAMS and integrated to Moodle. Int. J. Morphol., 29(2):363-370, 2011.

SUMMARY: This work relates to the experience of using LAMS in e-learning learning processes in the area of Health Sciences. This work was developed for the target subject matter of Human Liver Anatomy, following educational standards and the necessary methodologies to create Learning Designs (LD) and high-quality, interoperable, educational resources. The Institute of Anatomy, Histology, and Pathology was in charge of the design and implementation of the Learning Objects (LOs) in Liver Anatomy (using Macromedia Flash), while the Informatics Institute was in charge of the administration and processing of the pedagogical resource, integrating a specialist (LAMS and Moodle) for learning design and management. LAMS was used to create learning activities based on a Moodle integrated LD, as part of a medical students course. The satisfaction questionnaire applied to these students showed that nearly $70 \%$ of them considered the activities and objectives to be comprehensible and at the right level of detail, the evaluations to be coherent with the subject matter of the course, and in their opinion there was collaborative learning. Likewise, $67 \%$ assured that they would use a lesson plan in this modality again, and only $33 \%$ said they would do it occasionally.

KEY WORDS: Learning Designs; Learning Objects; Human Anatomy; LAMS; Moodle.

\section{REFERENCIAS BIBLIOGRÁFICAS}

Bucarey, S. \& Álvarez, L. Metodología de construcción de objetos de aprendizaje para la enseñanza de anatomía humana en cursos integrados. Int. J. Morphol., 24(3):357-62, 2006.

Bucarey, S. \& Álvarez, L. Metodología de Construcción de Objetos de Aprendizaje para la Enseñanza del Hígado Humano. Taller Internacional de Software Educativo, TISE. Santiago, Chile, 2004. pp. 42-8.

Bucarey, S.; Cabezas, X.; Vieira de Castro, A.; Araya, E.; Esperón, R.; Márquez, M. Manual de Construcción Colaborativa de Objetos de Aprendizaje para el Área de la Salud. Valdivia. Editorial Universidad Austral de Chile, Valdivia, 2010.

IEEE LOM. IEEE 1484.12.1-2002 Draft Standard for Learning Object Metadata. 2002. Available at http:// ltsc.ieee.org/wg12.

IMS Global Learning Consortium, Inc. IMS Learning Design Best Practice and Implementation Guide. Version 1.0 Final Specification, 2003.

Koper, R. \& Tattersall, C. (Eds.). Learning Design: A Handbook on Modelling and Delivering Networked Education and Training. Berlin Heildelberg, SpringerVerlag, 2005.

The Open Polytechnic of New Zealand. Technical Evaluation of selected Learning Management Systems. 2004. Available at: https://eduforge.org/docman/ view.php/7/18/LMS\%20Technical\%20Evaluation\%20\%20May04.pdf
Dirección para correspondencia

Sandra Bucarey A.

Instituto de Anatomía, Histología y Patología

Edifico de Cs. Biomédicas.

Campus Isla Teja

Universidad Austral de Chile

Valdivia

CHILE

Email: sbucarey@uach.cl

Recibido : 23-11-2010

Aceptado: 23-03-2011 